\title{
Cathodoluminescence Mapping of Defect Regions in Cadmium Sulfide Nanowires
}

\author{
Ovidiu Cretu ${ }^{1}$, Chao Zhang $^{1,2}$ and Dmitri Golberg ${ }^{1,3}$ \\ 1. International Center for Materials Nanoarchitectonics (MANA), National Institute for Materials \\ Science (NIMS), 1-1 Namiki, Tsukuba, Ibaraki 305-0044, Japan \\ 2. Graduate School of Pure and Applied Sciences, University of Tsukuba, 1-1-1 Tennodai, Tsukuba, \\ Ibaraki 305-8577, Japan \\ 3. Science and Engineering Faculty, Queensland University of Technology (QUT), 2 George St., \\ Brisbane, QLD 4000, Australia
}

The luminescence centers in cadmium sulfide (CdS) nanowires are mapped using a newly-developed system that allows the study of band-gap materials through cathodoluminescence experiments inside a high resolution transmission electron microscope. This is made possible by positioning an optical fiber within a few micrometers of the area of interest and scanning the focused electron beam while simultaneously collecting the generated photons. This technique is applied in order to characterize nanowires made from a well-known luminescent semiconductor - CdS.

The schematic layout of the system is illustrated in Figure 1a. A multi-mode optical fiber is mechanically connected to a piezoelectric tube, which allows for its positioning with sub-nanometer accuracy. A voltage source is connected to the scanning coils of the microscope and precisely controls the electron beam. The electrons interact with the sample and produce several signals: photons are collected by the optical fiber and transferred to a spectrometer, while scattered electrons are collected by an annular electron detector. Both these signals are acquired simultaneously and mapped across the region of interest.

A low-magnification image of a nanowire is shown in Figure 1b. It has a diameter of around $100 \mathrm{~nm}$ and a length of several micrometers, which is typical of the nanowires that were tested. High-resolution images and selected-area diffraction patterns confirm that these structures are single crystals, corresponding to the wurtzite structure.

The result of one of the experiments is summarized in the lower part of Figure 1. Figure 1c shows the simultaneously-acquired dark-field image of the $200 \mathrm{~nm}$-wide nanowire. In this particular case, the data is acquired from the last $1 \mu \mathrm{m}$-long section of the wire, which ends in the lower part of the image. Figure 1d depicts the cathodoluminescence data-cube acquired form the same region.

Our results show that, while some defects are already present as a result of the growth process, many of the defects responsible for luminescence are produced as a result of electron irradiation damage. The electron beam creates defects corresponding to two regions: between 500 and $550 \mathrm{~nm}$, which we assign to $\mathrm{S}$ interstitials, and between 650 and $800 \mathrm{~nm}$, which we attribute to $\mathrm{S}$ vacancies. The way in which these peaks are distributed is also different: the first group features localized maxima, while the second group is distributed uniformly across the surface of the sample. These defects are a by-product of the same damage process.

We have used a combination of control experiments, electron damage calculations and literature review in order to assign several of the observed peaks to specific defect structures. Other interesting results are 
expected by applying the same technique on analogous semiconducting nanomaterials [1].

\section{References:}

[1] The authors would like to thank Dr. Martin Elborg and Dr. Yoshio Bando (NIMS). Funding was provided by the National Institute for Materials Science (NIMS) through the International Center for Materials Nanoarchitectonics (MANA). D.G. also acknowledges Grant-in-Aid No. 26289244 (MEXT, Japan). O.C. is grateful to the International Center for Young Scientists (ICYS) of NIMS for financial support.

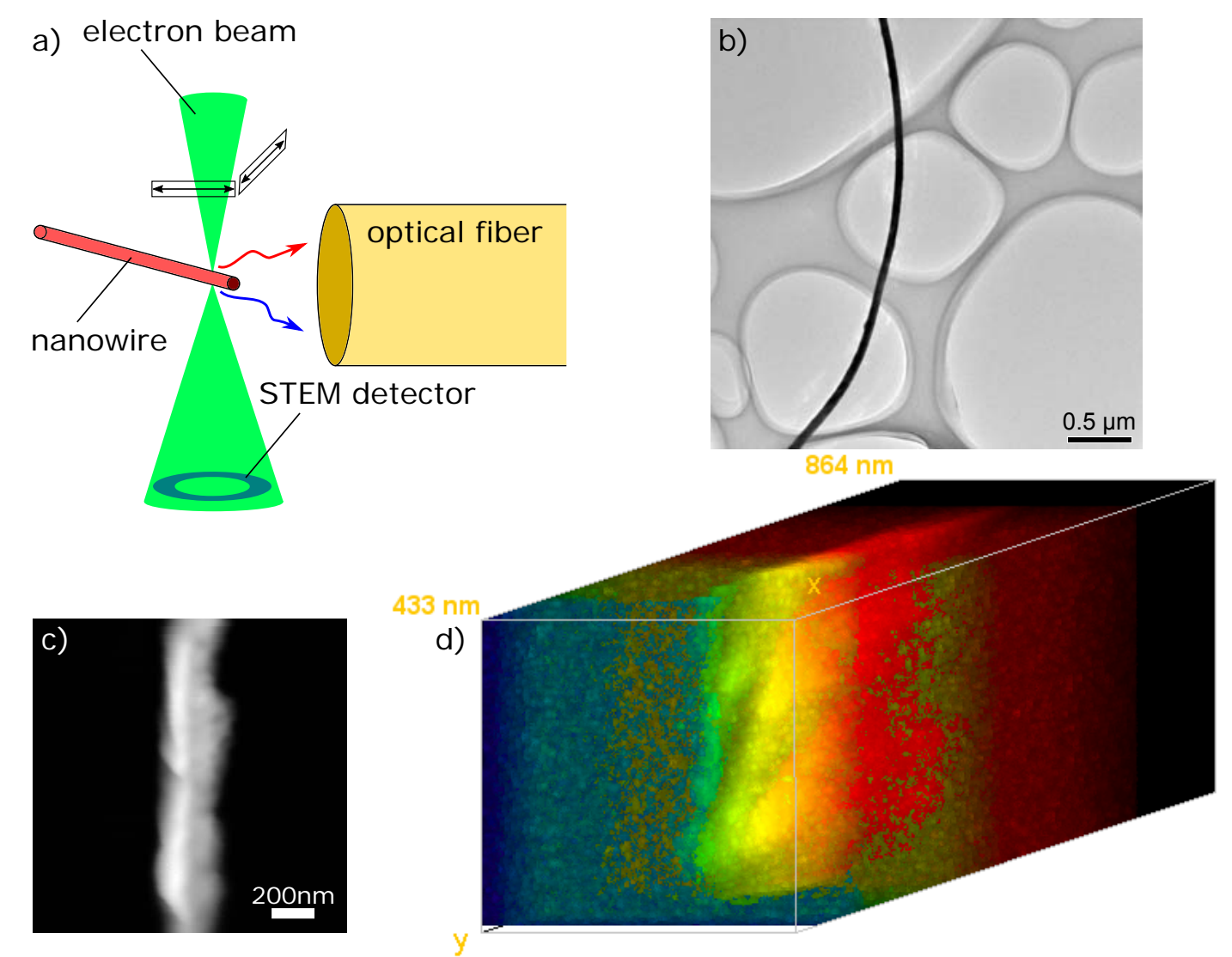

Figure 1. a) Schematic of the experimental set-up. b) Representative image of a typical CdS nanowire. c) ADF-STEM image acquired simultaneously from the nanowire region of interest. d) CL data-cube of the area displayed in c). 